\title{
Evaluación de objetos digitales de aprendizaje musical en Moodle
}

\section{Assessment of digital learning objects in Moodle}

\author{
Manuel Jesús Espigares Pinazo*1 \\ pmje79@gmail.com \\ José Manuel Bautista Vallejo** \\ josembau@gmail.com \\ *Universidad Internacional de La Rioja, España \\ **Universidad de Huelva, España
}

\section{Resumen:}

Este estudio presenta la aplicación de procesos automatizados de análisis a objetos digitales de aprendizaje musical online, mediados por una plataforma telemática. A nivel teórico, se basa en la aplicación de los principios del aprendizaje virtual, la educación personalizada y la aplicación de procesos automatizados de análisis de datos educativos. En concreto, se plantea la aplicación de dichas técnicas a los test de evaluación inicial, para medir niveles de conocimientos previos en la materia. El análisis de la información se efectúa a partir de los datos recogidos en una herramienta para la elaboración de cursos online, Moodle. A partir de dichos datos se halla un modelo, denominado k-medias ( $k$-means en lengua inglesa), que permite clasificar los diferentes niveles de conocimientos musicales. El modelo elaborado establece tres perfiles en cuanto al nivel de aprendizaje musical del alumnado: nivel alto, medio y bajo.

\section{Palabras clave:}

TIC; Moodle; objetos digitales de aprendizaje; Educación Musical; Software Libre; Recursos Telemáticos.

\begin{abstract}
:
This study presents the application of automated analysis processes to digital objects in online music learning, mediated through a telematic platform. TheoreticaIly, the study is based on the application of the principles of e-learning, personalized education and implementation of automated processes for analysing educational data. Specifically, this study taps into the application of such techniques to initial test assessment, with a view to measuring levels of subject knowledge. The analysis of the information is undertaken from the data collected in a tool for making online courses, Moodle. From the analysis of these data a model, called k-means, emerges which classifies the different levels of musical knowledge. The model establishes three profiles regarding acquisition of music knowledge: high, medium and low.
\end{abstract}

\section{Keywords:}

ICT; Moodle; learning object; musical education; free software; telematic resources.

1 Dirección para correspondencia (correspondence address):

Manuel Jesús Espigares Pinazo. Departamento de Didáctica. Universidad Internacional de La Rioja. C/Hermenegildo, 2. Dos Hermanas, Sevilla (España). 


\section{Résumé:}

Cette étude présente l'application de processus automatisés d'analyse aux objets numériques d'apprentissage musical en ligne, médiatisés par une plate-forme télématique. Sur le plan théorique, il repose sur l'application des principes de l'apprentissage virtuel, de l'éducation personnalisée et de l'application de processus automatisés d'analyse des données éducatives. Plus précisément, l'application de ces techniques aux tests d'évaluation initiaux est proposée, afin de mesurer les niveaux de connaissances antérieures sur le sujet. L'analyse de l'information est faite à partir des données recueillies dans un outil pour le développement de cours en ligne, Moodle. À partir de ces données, il existe un modèle appelé k-means (anglais k-means), qui permet de classer les différents niveaux de connaissances musicales. Le modèle élaboré établit trois profils en termes de niveau d'apprentissage musical des étudiants: niveau élevé, moyen et faible.

\section{Mots clés:}

TIC; Moodle; objets d'apprentissage numérique; éducation musicale; logiciels libres; ressources télématiques.

Fecha de recepción: 4-10-2017

Fecha de aceptación: 21-12-2017

\section{Introducción}

La presente investigación se ubica en el campo de la telemática aplicada a la Educación Musical (unión entre informática, telecomunicaciones, educación y música) aplicada a la etapa de la Educación Secundaria Obligatoria. Debido a la cada vez más elevada presencia de la tecnología digital en los procesos de enseñanza-aprendizaje musical, se plantea el desarrollo de esta línea de estudio como una necesidad dentro del ámbito de las ciencias de la educación. Las aplicaciones y recursos online son cada vez mayores (Spector, 2013; Cano, 2016; Mullins, Hicks, Ogle, Schilder y van Hover, 2016,). Por ello, y debido a las nuevas necesidades y retos que se plantean en la actual sociedad digital de la información, se presenta un uso concreto de una plataforma telemática que favorece un elevado nivel de control del proceso de enseñanza-aprendizaje del alumnado y algunas variables como, por ejemplo, las puntuaciones en los test para evaluar el nivel de conocimientos del alumnado, las cuales quedan registradas y permiten su posterior análisis y estudio detallado.

En este marco, el estudio que se presenta permite, en el ámbito de la tecnología, atender a la necesidad de evaluar las evaluaciones iniciales que el alumnado realiza a principio de curso, a través de herramientas basadas en procesos de enseñanza-aprendizaje mediados por las TIC, en concreto mediante el empleo de plataformas telemáticas y la informa- 
ción recogida en las bases de datos de dichos soportes informáticos, en concreto el sistema de gestión del conocimiento (Learning Management System o LMS) Moodle. La herramienta empleada en el estudio como soporte para la recogida y análisis de datos, Moodle, es el acrónimo de Modular Object-Oriented Dynamic Learning Environment. Se trata de un software libre para la realización de cursos masivos en línea (en inglés, MOOC o Massive Open Online Courses). Además Moodle, como es sobradamente conocido, es un software para producir cursos basados en el uso de Internet, además de páginas web. Por otro lado, es un proyecto desarrollado para su libre distribución y para apoyar el constructivismo social dentro del marco educativo.

Dicha herramienta, Moodle, incluye diferentes objetos digitales de aprendizaje tales como chats, foros, talleres online, tareas basadas en las TIC y herramientas de comunicación que se definen como objetos digitales de aprendizaje (Chan, Galeana y Ramírez, 2007; Jorgensen, 2008; Rivera, 2014). Tal y como señalan estos autores, algunas de las características más destacables de este tipo de objetos son su capacidad de adaptación, polivalencia y las posibilidades que ofrecen como herramientas de comunicación en los contextos de enseñanza aprendizaje mediados por las TIC.

\section{Marco teórico}

Numerosos estudios han descrito variadas ventajas de la plataforma Moodle y el uso de los objetos digitales de aprendizaje, al igual que también se han analizado algunos de sus mitos (Selwyn, 2012; Bullock, 2016). Algunas de las ventajas de esta plataforma a las que se refieren los estudios son (Aydin y Tirkes, 2010; Kop, 2011; Kearney y Levine, 2015): apoya la pedagogía social constructivista (colaboración, actividades de aprendizaje, reflexión crítica, etc.), es apropiada para la educación presencial y a distancia, presenta una interfaz compatible con cualquier navegador, es fácil de instalar en la mayoría de las plataformas (incluidos smartphones o tabletas), de fácil administración: el alumnado puede crear sus propias cuentas, un perfil en línea incluyendo sus fotos y su descripción, intereses, gustos y motivaciones, elegir el formato del curso por semana, por tema o por tema de discusión basado en un formato social que potencia la comunicación y el intercambio de información en 
procesos de enseñanza-aprendizaje musical (Himonides y King, 2014; Montgomery, 2015). También podemos incluir las ventajas en relación a cómo el alumnado comparte conocimiento y discute sobre los distintos temas, las cuales han sido estudiadas por Yang, Zhang, Su y Tsai (2011).

Dentro de este contexto de trabajo, que aplica procedimientos de análisis automatizados en una plataforma telemática para el aprendizaje musical virtual, se arrojan perfiles de los usuarios y se presta una especial atención a la educación personalizada. Entre las ventajas más destacables de este modelo de trabajo está el poder gestionar la totalidad de los datos registrados sobre aprendizaje y establecer perfiles del alumnado mediante pruebas de diagnóstico o evaluaciones iniciales, realizadas al principio del curso académico por parte de todos los centros educativos. El hecho de atender a la educación personalizada supone, desde el principio del proceso de enseñanza-aprendizaje atender a los intereses y motivaciones del alumnado. Teniendo, de esta forma, en cuenta las características individuales de cada uno de ellos, monitorizando su trabajo y orientando toda de su labor de forma correcta. En esta línea, Bernardo (2014), señala que la educación personalizada supone una herramienta útil para el presente y el futuro de la educación. Atiende a lo que las personas tienen en común o comparten (su propia naturaleza) y el hecho de ser diferentes, sus principios y fundamentos. También aúna factores como la socialización y la individualización educativas y constituye el tipo de educación más apropiada para la sociedad digital en la que estamos inmersos. Además potencia la adquisición de las competencias clave de forma que nos encaminemos de manera libre y responsable hacia el control de nuestras propias vidas, el desarrollo de la autonomía personal y el autoaprendizaje.

En este sentido, en este estudio se emplea una plataforma educativa, que en resumen plantea la creación de una comunidad virtual de aprendizaje (CVA). En esta línea, Coll y Monereo (2008) y Monedero-Moya, Cebrián-Robles y Desenne (2015) apuntan que gracias a las TIC, estas comunidades de aprendizaje se conforman independientemente de las barreras físico-temporales. Cuthbert (2003) indica que una comunidad de aprendizaje (CA), favorece la comprensión y desarrolla un sistema común de criterios para la evaluación de ideas.

Dentro de los campos de estudio vinculados con el uso de Moodle y los objetos en objetos digitales de aprendizaje destinados a la formación online, cabe añadir que, los procesos automatizados de análisis se 
plantean como una solución adecuada el análisis de datos recogidos en Moodle. Este campo, presenta el uso de aplicar el uso y desarrollo de procesos automatizados de análisis a partir de modelos estadísticos a partir de grandes volúmenes de información (como la que se genera hoy en día con los cursos masivos en línea) (Bizer et al., 2011; Lin et al., 2013), aporta información detallada sobre el funcionamiento de los agentes educativos de estas comunidades (participación de padres, madres, profesorado, alumnado e instituciones educativas (Etscheidt y Curran, 2012) y de los objetos de aprendizaje (herramientas de comunicación como chats, foros, test de autoevaluación y actividades colaborativas) en el marco de las plataformas de teleformación o sistemas de gestión virtual de los aprendizajes (LMS o learning management systems). Tal y como señalan Chan, Galeana y Ramírez (2007), las plataformas de teleformación poseen virtudes que permiten gestionar de manera óptima los objetos de aprendizaje virtual, ya que aglutinan todo tipo de herramientas para configurar y manipular dichos objetos de aprendizaje variados, con un alto grado de elaboración, adecuación y sofisticación.

En cuanto a la evaluación del alumnado en la plataforma empleada, Moodle, autores como Valverde, Revuelta y Fernández (2012) proponen su uso como herramienta de gestión del conocimiento y debido a sus posibilidades a la hora de ofrecer un feedback inmediato del trabajo del alumnado. También permite centralizar la información y trabajar con rúbricas que pueden ser ponderadas a través de las diferentes herramientas de evaluación con las que cuenta la propia plataforma telemática.

La plataforma Moodle sirve como soporte para la elaboración de modelos estadísticos y su posterior análisis. De cara a la clasificación de la información, uno de los modelos que resultan útiles es el denominado $K$ medias, que agrupa conjuntos de datos en grupos distintos (o conglomerados) (Celebi, Kingravi y Vela, 2013). El método define un número fijo de conglomerados, de forma iterativa asigna registros a los conglomerados y ajusta los centros de los conglomerados hasta que no se pueda mejorar el modelo (Lin et al., 2012), como veremos, más adelante en la Figura 3. En lugar de intentar pronosticar un resultado, los modelos de Kmedias utilizan un proceso conocido como aprendizaje no supervisado (Feldman, Schmidt y Sohler, 2013) para revelar los patrones del conjunto de campos de entrada. Lo cual va a resultar útil para el establecimiento de perfiles o niveles de aprendizaje.

Según Irigoien y Arenas (2006, 261) el análisis cluster, o clustering, 
"es una colección de métodos estadísticos que permiten agrupar casos sobre los cuales se miden variables o características". En este sentido, el clasificar objetos es una necesidad y algo muy habitual en el mundo actual, ya que permite ordenar y agrupar para conocer las categorías en las que se puede constituir la propia realidad o los objetos estudiados. Los clusters o grupos que se forman a través del análisis K-medias, tienen la peculiaridad de basarse en que no contamos con información previa y se sugieren a partir de la propia esencia de los datos. Para la elaboración del K-medias existen una gran cantidad de técnicas, algunas de ellas muy complejas (Lin et al., 2012). Una de las características del K-medias es que es un método de análisis cluster no jerárquico y que la cantidad de clusteres que se van a formar son fijados a priori. A partir de ahí, los objetos se agrupan para obtener dichos conjuntos con algún criterio de optimización. En cuanto a la naturaleza de los datos empleados, esta técnica emplea variables cuantitativas y es ideal para analizar grandes volúmenes de datos o información recogida en procesos de enseñanzaaprendizaje mediados por plataformas telemáticas (Mairal et al., 2010), tal como el caso que presentamos.

Este modelo K-medias, además, se presenta como una solución ideal, fácil y sencilla de utilizar, en los cursos masivos en línea (MOOC), gracias a sus posibilidades de automatización y configuración (Kay et al., 2013), siendo un método eficaz y eficiente para el conocimiento de los niveles de rendimiento académico del alumnado (Redding, 2014a; Redding, 2014b; Sagitova, 2014). De esta forma se atiende de manera rigurosa y objetiva a la educación personalizada (RAND Corporation, 2014; Twyman, 2014), mediante el análisis de los datos obtenidos a partir de las pruebas de evaluación final. El algoritmo K-medias empleado, en definitiva, conecta directamente con las bases de datos de la plataforma de aprendizaje y permite ser programado (Mairal et al., 2010; Lin et al., 2012), para recibir información actualizada y automatizada, de los niveles de adquisición de competencias musicales del alumnado.

En resumen, el estudio que se presenta expone un método simplificado para el trabajo del profesorado con esta técnica estadística, permitiendo manejar de manera sencilla, de forma automatizada, datos en tiempo real y organizar en categorías o grupos dicha información para después ser sometida a estudio y tomar decisiones educativas. 


\section{Objetivo del estudio}

El objetivo del estudio es demostrar la utilidad de las técnicas automatizadas de análisis de objetos digitales, para el análisis de datos educativos musicales en torno a la evaluación inicial de los distintos perfiles de rendimiento escolar: estableciendo un perfil bajo, medio y alto. En concreto se presenta un modelo tipo cluster denominado K-medias. Dicho modelo, tal y como se indica en el marco teórico, se obtiene a partir de un sistema automatizado de análisis de datos educativos, el cual, posibilita la recogida de datos y su posterior modelización.

\section{Método}

Se realiza un estudio basado en una metodología cuantitativa. Los datos de los test respondidos por el alumnado, son procesados en tiempo real y a través de un algoritmo de análisis de datos que permite la elaboración de un modelo estadístico que agrupa y organiza la información de forma estructurada, con un criterio pedagógico, didáctico y educativo.

\subsection{Participantes}

Para este estudio sobre la eficiencia y utilidad del uso de las técnicas y procedimientos automatizadas de análisis, en los procesos de enseñanza-aprendizaje musical en Moodle, mediados por los MOOC, 50 centros de España $(n=50)$ han sido invitados a participar de forma totalmente confidencial y anónima. La invitación ha sido realizada mediante el uso del correo electrónico de los diferentes centros educativos.

La muestra del estudio, en el que han participado 50 centros educativos a nivel nacional, es de 1345 estudiantes de primero de la ESO con edades comprendidas entre los 12 y 13 años.

\subsection{Instrumento}

Se emplea para la recogida de datos un test consistente en 7 preguntas para medir los perfiles del alumnado: alto, medio y bajo, en concreto, valorando de esta forma la competencia básica o clave denominada cultural y artística del alumnado de $1 .^{\circ}$ de la ESO. 
Las cuestiones abordan diferentes bloques temáticos como fundamentos del lenguaje musical, diferenciación entre signos musicales, clasificación de voces e instrumentos musicales, música y TIC, distinción entre sonidos en diferentes claves musicales o audición musical. Las preguntas específicas del test aparecen en el anexo de este trabajo y pueden ser consultadas.

\subsection{Procedimiento}

La recogida de datos se realizó durante las dos últimas semanas del curso 2014-2015. En concreto, como referimos anteriormente, se estudian los tests de evaluación final, realizados a final del curso académico para medir el nivel de adquisición de conocimientos musicales del alumnado. El test ha sido respondido de forma totalmente confidencial y anónima a través de la modalidad online. Los centros que han participado en el estudio han sido elegidos de forma aleatoria y a través del correo electrónico, se les ha solicitado la participación en la investigación, como hemos señalado anteriormente.

Como material para la elaboración del estudio se emplea una plataforma telemática Moodle alojada en un servidor informático y el software de modelización estadística Clementine, en su versión 11.1.

Los análisis han sido efectuados con el programa SPSS Clementine 11.1, programa especializado en el desarrollo de técnicas automatizadas de análisis) y la elaboración de modelos estadísticos. Dicho software ha sido conectado a la base de datos de la plataforma telemática Moodle. Esto ha permitido la monitorización de los test y la obtención de informes detallados sobre la realización del mismo. Se ha elaborado un proyecto que permita automatizar tareas de análisis como la obtención de información y la exportación de resultados al sistema utilizado para trabajar, la plataforma de teleformación Moodle y su base de datos.

El procedimiento de análisis del estudio consta en total de 3 fases:

1. Técnica exploratoria aplicada mediante el Modelo cluster K-medias.

2. Definición de un criterio pedagógico que establezca los distintos perfiles de rendimiento escolar: alto, medio y bajo, partiendo del agrupamiento realizado mediante modelo cluster exploratorio.

3. Aplicación de una fórmula a la totalidad de los datos del test, a partir del modelo cluster y los rangos establecidos en torno a los perfiles de rendimiento del alumnado. 
En primer lugar se realiza la exploración de la información y elaboración del modelo cluster. En este apartado se reflejan los análisis efectuados con SPSS.

La elaboración de los análisis que se muestra en este apartado del estudio se basa en un trabajo dividido en con tres fases, perfectamente delimitadas. En primer lugar, cuando se trabaja con técnicas automatizadas de análisis, se somete a los datos a una depuración y selección de la información. En concreto, se seleccionan las variables o variable objeto de estudio, en concreto las puntuaciones de los test de evaluación inicial y eliminamos los valores nulos, que no sirven para la obtención del modelo tipo cluster. A continuación, en una segunda fase se aplica la técnica del K-medias y se selecciona el número de conglomerados que queremos obtener. En el presente artículo, al dividir los grupos del alumnado en tres perfiles en cuanto a su nivel de conocimientos previos musicales: bajo, medio y alto. De esta forma el espacio de cada conglomerado aparecerá delimitado por este criterio de clasificación y mostrará un índice de distancia con respecto al centroide o el valor en torno al cual se van agrupar los datos de cada grupo (como veremos en la gráfica, más adelante). En tercer lugar, para esclarecer y comprender mejor el modelo obtenido se presentan en una gráfica los tres conglomerados obtenidos y agrupados por el algoritmo k-medias empleado.

La Figura 1 muestra, de forma general, el proyecto de análisis automatizado elaborado para la obtención del modelo K-medias en este estudio. Se observa la posibilidad de programar de forma gráfica, algoritmos que permiten la automatización de los procesos de análisis. Los datos se pueden dividir en particiones, esto sería interesante, sobre todo, cuando se manejan grandes volúmenes de información, registros y variables, e incluso emplear técnicas de muestreo con datos escogidos de forma aleatoria y entrenar el modelo para que sea lo más preciso posible. Una vez entrenado el modelo, podría aplicarse al total de la muestra y de forma automática y en tiempo real, obtener los resultados de los análisis a partir de los datos de la base de datos (o bases de datos, en el caso de que se empleen varias) de la aplicación informática que se utiliza para la recogida de la información. 


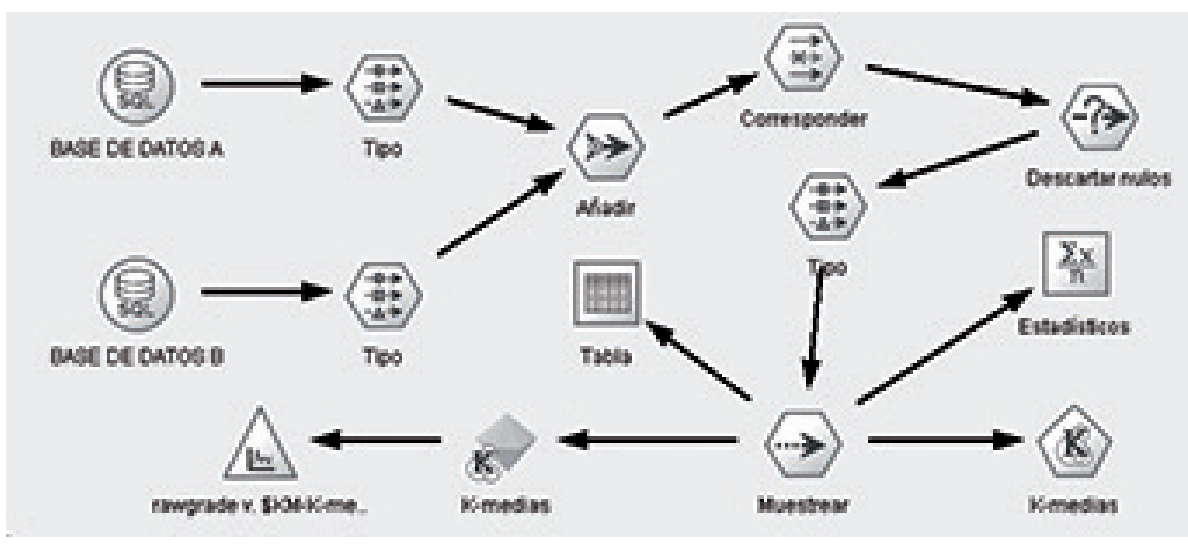

Figura 1. Proyecto de análisis de datos basado en las técnicas automatizadas de análisis.

Después de observar cómo sería un proyecto basado en el empleo de técnicas automatizadas de análisis, se muestra una tabla con los estadísticos básicos y la distribución de la muestra. La tabla muestra un recuento de registros válidos de 1345 en total, es decir que dicho número se corresponde con los alumnos y alumnas identificados con un "id", es decir, un número identificador único que se vincula con su nombre y datos personales de la base de datos del sistema, que permite conocer en todo momento de qué alumnos se está hablando y monitorizar su trabajo hacer como hacer el seguimiento de su proceso de enseñanzaaprendizaje.

La media de las puntuaciones de los test de evaluación inicial para medir el nivel de conocimientos musicales previos es 78,845 en una escala de 0 a 100. El mínimo es 0 y el máximo 100. La varianza 382,154 y la desviación típica 19,549. La mediana 80,000 y la moda 100.

Seguidamente, se muestra la elaboración del modelo K-medias paso a paso y las imágenes que clarifican los resultados obtenidos en el trabajo. En el modelo obtenido en el estudio, se pueden ver 3 bloques, conglomerados o clusters. Cada uno de estos clusters presenta niveles de conocimientos musicales previos, obtenidos a partir de las evaluaciones iniciales, que el alumnado realiza al principio del curso académico:

1. El conglomerado 1 presenta una muestra de 577 registros. La media es 65,03 en una escala de 0 a 100. También hemos establecido índices de proximidad de unos clusters con respecto a otros. La desviación típica es de 9,061. El índice de proximidad del cluster 1 con el cluster 3 es 
de 0,28996 y del cluster 1 con respecto al cluster 2 es 0,405908. Este cluster aparece definido por el grupo formado por un alumnado con un nivel medio de conocimientos musicales previos.

2. El conglomerado 2 refleja una muestra de 49 registros. La media es 24,439 en una escala de 0 a 100. La desviación típica es 11,71. Los índices de proximidad del cluster 2 con respecto al cluster 1 es de 0,405908. El cluster 2 con respecto a cluster 3 es de 0,695868. El cluster 2 representa al alumnado con un nivel bajo de conocimientos musicales previos.

3. El conglomerado 3 arroja un total de 709 registros. La media es 94,026 en una escala de 0 a 100. La desviación típica es 6,708. El índice de proximidad del con respecto al cluster 1 es 0,28996 y el cluster 2 0,695868. El cluster 3 representa al alumnado con un nivel alto de conocimientos musicales previos.

A continuación, en la Figura 2, se presenta el modelo K-medias de forma gráfica, con un alto grado de fiabilidad y que arroja como valor 1,00 . También observamos el gráfico de sectores y de barras la distribución de la muestra del modelo expuesto con los conglomerados que establecen los niveles de conocimientos musicales previos.

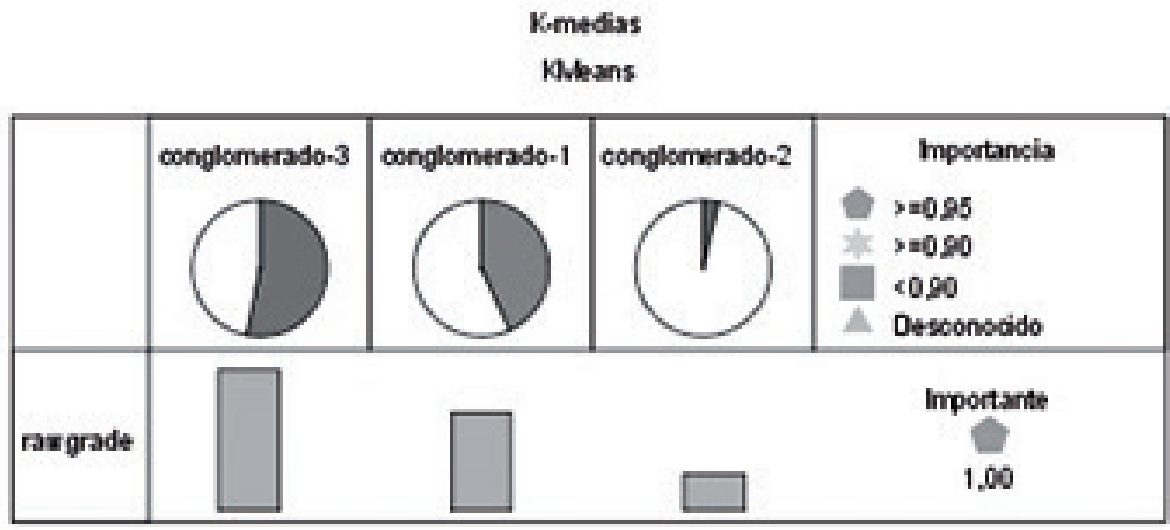

Figura 2. Modelo K-medias generado que representa la alta fiabilidad del modelo obtenido y las gráficas de sectores y barras con la distribución de la muestra en los niveles de conocimientos previos: alto, medio y bajo.

Seguidamente aparece la gráfica (Figura 3) con el modelo, correspondiente a las puntuaciones obtenidas y los diferentes conglomerados. En la imagen se muestra con claridad los tres niveles que planteamos en nuestro marco teórico: alto, medio y bajo. Dichos niveles serán los 
niveles de conocimientos previos del alumnado que participa de forma anónima en el estudio.

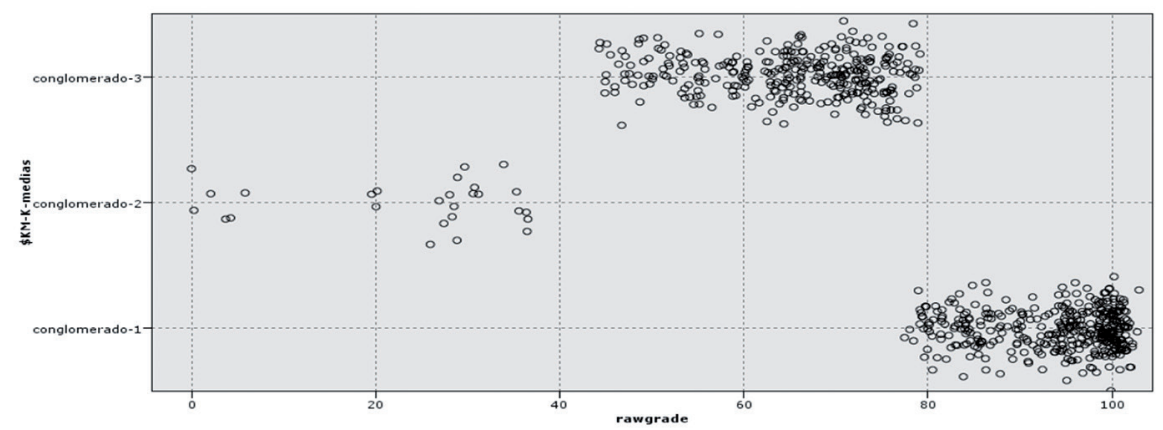

Figura 3. Modelo K-medias obtenido con los tres niveles de conocimientos previos del alumnado, obtenidos a partir de los test de evaluación inicial.

En la siguiente figura (Figura 4), se observa cómo están distribuidos de forma esquemática y partiendo del gráfico con el análisis K-medias anterior, (donde se aprecian los conglomerados correspondientes a los distintos perfiles de alumnado), la distribución de los agrupamientos a partir de los datos recogidos tras la realización de las evaluaciones iniciales. Los perfiles quedan establecidos de la siguiente manera: nivel alto (8-10 puntos), nivel medio (5-7 puntos) y nivel bajo (0-4).

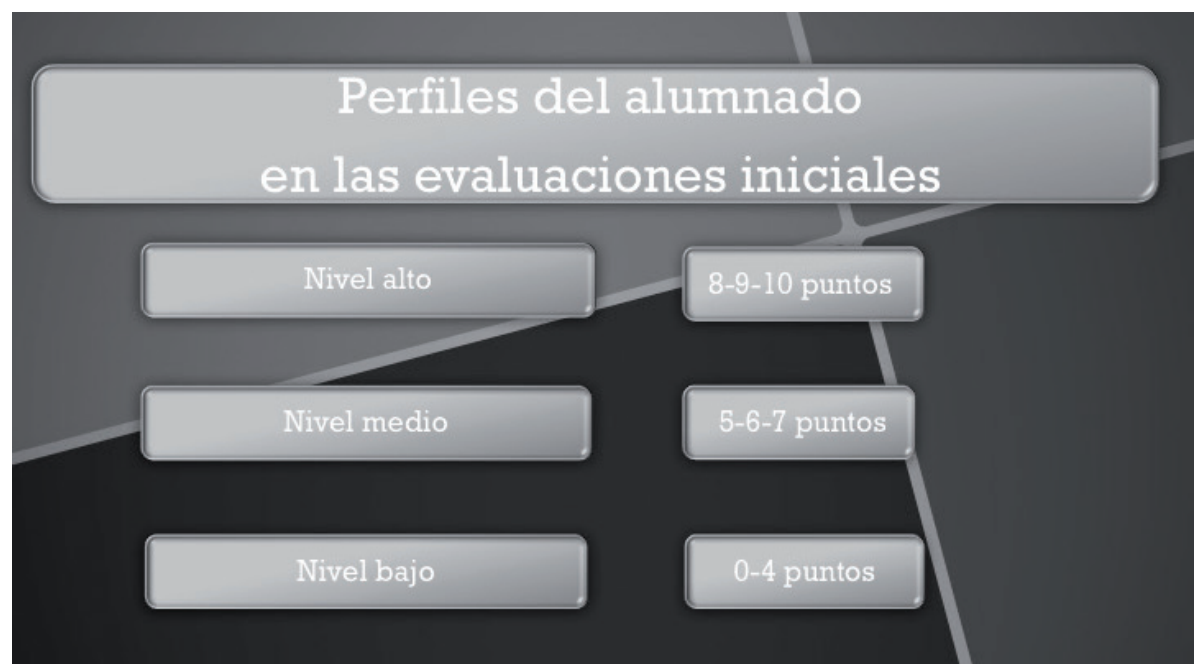

Figura 4. Perfiles del alumnado tras la realización de las evaluaciones iniciales y el análisis cluster. 
A continuación, en el siguiente apartado, se muestran las conclusiones del estudio sobre elaboración de un modelo K-medias, a partir de las evaluaciones iniciales, efectuadas a principio de curso y que establecen los niveles de conocimientos musicales previos del alumnado, a través de perfiles: alto, medio y bajo.

\section{Conclusiones y prospectivas}

Los resultados que se obtienen en el siguiente artículo son:

1. En primer lugar, mediante las técnicas automatizadas de análisis de datos educativos se puede monitorizar y establecer niveles previos de aprendizaje musical. En concreto en este estudio, el Modelo K-medias, que se ha configurado para la elaboración de 3 clusters diferentes, ha permitido delimitar un nivel de conocimientos musicales altos, medios y bajos. Esto posibilita potenciar la educación personalizada en la escuela y atender a la diversidad educativa en el aula de forma útil y eficaz. Los clusters obtenidos muestran un alto índice de alumnado con un nivel de conocimientos musicales previos y un bajo número de alumnado con nivel de conocimientos musicales a la hora de realizar el test de evaluación inicial.

2. El establecimiento de diferentes niveles de aprendizaje permite desarrollar actividades de aprendizaje adaptadas con diferentes niveles de dificultad y adaptadas a las necesidades particulares, intereses y motivaciones del alumnado.

3. El estudio realizado, ha permitido monitorizar la realización del test de evaluación inicial, en cuanto a su cumplimentación y realización del mismo, en cualquier momento a lo largo de la investigación. A través del empleo de las técnicas automatizadas de análisis en educación con Moodle, se posibilita tener un alto grado de control de los datos recogidos y hacer todo tipo de adaptaciones en tiempo real. Dichas adaptaciones, se realizan tanto en los procesos de recogida de información como en su posterior análisis de resultados obtenidos.

4. A partir de la naturaleza o tipología de los datos recogidos, los proyectos de análisis de datos educativos basados en el empleo de procesos analíticos automatizados permiten redefinir los modelos utilizados, ya sean de exploración, descriptivos, de clasificación y predictivos. En este sentido se ofrece un amplio de abanico de posibilidades de análisis y monitorización de la información. 
5. El empleo de procesos automatizados de análisis en los MOOC o cursos online masivos, es decir destinados a un elevado número de estudiantes, permite de manera rápida y personalizada, adaptar los procesos de análisis de datos educativos para tomar decisiones educativas, adecuadas a los diferentes niveles de aprendizaje en el aula. Estableciendo itinerarios de aprendizaje, a partir de los estilos de navegación vía web y las estrategias de aprendizaje, así como las medidas de atención educativa eficaces y adaptadas a las necesidades tanto individuales como colectivas del alumnado.

6. Las técnicas automatizadas de análisis presentan un alto nivel de idoneidad para el trabajo con cursos masivos en línea. Su enorme versatilidad, flexibilidad y potencia a la hora de trabajar con grandes volúmenes de datos las convierten en un recurso útil y eficiente, al servicio de la investigación educativa y el estudio de los procesos de enseñanzaaprendizaje online.

7. El modelo hallado, dada su fiabilidad del $100 \%$ supone que es escalable e independientemente del tamaño muestral, se puede aplicar a un sistema de análisis automatizado. Además, el modelo hallado es reproducible y repetible. De esta forma atendemos a dos principios básicos del método científico: reproducibilidad y repetibilidad, es decir, permite que sea aplicable a otros contextos de investigación en los que haya que clasificar la información del alumnado en perfiles de rendimiento académico: alto, medio o bajo.

8. En conclusión, el presente artículo ofrece una solución rápida y sencilla de un problema recurrente en las aulas de secundaria. Nos referimos al establecimiento de niveles educativos a partir de los test de evaluación inicial, que se realizan en todos los centros de enseñanza a principio de curso. Mediante el empleo de técnicas estadísticas avanzadas y análisis automatizados, procedentes de procesos automatizados de análisis (k-medias, modelos clusters o de conglomerado) aplicadas a los procesos de enseñanza-aprendizaje musical online (en los MOOC o cursos masivos en línea). Aquí, se presenta una forma eficaz, útil y novedosa, así como objetiva de establecer los niveles de aprendizaje, promoviendo la educación personalizada y la atención a la diversidad del alumnado.

En cuanto a las limitaciones y las prospectivas del trabajo, sería interesante de cara al futuro desarrollar y conseguir un mayor grado de integración de las herramientas de análisis de datos educativos en los 
MOOC de cara la evaluación de objetos digitales de aprendizaje. De esta forma, las herramientas para el aprendizaje, las plataformas telemáticas de código abierto como Moodle (entre otras) ofrecerían al profesorado de forma rápida y sencilla información detallada de los procesos de enseñanza-aprendizaje en la sociedad digital. Por ello, una de las necesidades, es trabajar en el desarrollo de modelos estadísticos adecuados a la tipología de actividades que desarrollemos, atendiendo a la tipología y la naturaleza de los datos recogidos.

Otras de las grandes líneas de estudio es el Big Data. Debido a que para aplicar este tipo de técnicas se necesitan grandes volúmenes datos, Moodle y los objetos digitales de aprendizaje online que incluye (foros, chats, cuestionarios, test, etc.) aportan un recurso adecuado para trabajar en este sentido. Estos procedimientos de análisis se hallan orientados a la mejora de la eficiencia de los sistemas, así como su utilidad como herramienta para el trabajo docente y el quehacer diario del profesorado.

Por último, cabe destacar que otra de las prospectivas de la investigación consiste en el trabajo con redes sociales. En concreto, la realización de actividades online y la monitorización de los procesos educativos en dichas redes digitales, así como la elaboración de modelos estadísticos válidos y fiables, útiles en tareas docentes, de investigación y gestión académica.

\section{Referencias}

Aydin, C.C., Tirkes, G. (2010). Open Source Learning Management Systems in Distance Learning. TOJET: The Turkish Online Journal of Educational Technology, 9(2), 175184.

Bernardo, J.B. et al. (2014). Educación personalizada: principios, técnicas y recursos. Logroño: Síntesis.

Bullock, S. (2016). Digital technologies in teacher education. From mythologies to making. In Kosnik, C.; White, S.; Beck, C.; Marshall, B.; Goodwin, A. Lin \& Murray, J. (eds.), Building Bridges. Rethinking Literacy Teacher Education in a Digital Era (pp. 2-16). Rotterdam: Sense Publishers.

Calderero Hernández, J.F. et al. (2014). Una nueva aproximación al concepto de educación personalizada y su relación con las TIC. Teoría de la Educación. Educación y Cultura en la Sociedad de la Información, 15(2), 131-151.

Celebi, M.E., Kingravi, H.A. \& Vela, P.A. (2013). A comparative study of efficient initialization methods for the k-means clustering algorithm. Expert Systems with Applications, 40(1), January, 200-210. 
Evaluación de objetos digitales de aprendizaje musical en Moodle Manuel Jesús Espigares Pinazo y José Manuel Bautista Vallejo

Chiappe-Lavarde, A., Hine, N. \& Martínez-Silva, J.-A. (2015). Literatura y práctica: una revisión crítica acerca de los MOOC. Comunicar, 44 (XXII), 9-18. DOI http://dx.doi. org/10.3916/C44-2015-01G

Coll, C. y Monereo, C. (2008). Psicología de la educación virtual. Madrid: Morata.

Cuthbert, A.J. (2003). Wise learning Communities. Design Considerations. In A.K. Renninger y W. Shumar (Eds.), Building Learning communities. Learning and change in cyberspace (pp. 215-246). Cambridge: Cambridge University Press.

Chan, M.E., Galeana, L. y Ramírez, M.S. (2007). Desarrollo de objetos de aprendizaje basado en patrones. Brasil: Virtual Educa.

Estadística (2016). Portal de la Universidad de Granada sobre estadística.

Recuperado el 12 de Enero de 2016 de http://wdb.ugr.es/ bioestad/guia-spss/practica-8/\#2

Etscheidt, S. \& Curran, C. (2012). Promoting Reflection in Teacher Preparation Programs: A Multilevel Model. Teacher Education and Special Education, 35(1), 7-26. (http:// doi.org/dk 53×2).

Feldman, D., Schmidt, M. \& Sohler, C. (2013). Turning big data into tiny data: constantsize coresets for k-means, PCA and projective clustering. SODA'13 Proceedings of the Twenty-Fourth Annual ACM-SIAM Symposium on Discrete Algorithms, New Orleans, Louisiana, pp. 1434-1453.

Himonides, E \& King, A. (2014). Proceedings of the Sempre MET2014: Researching Music, Education, Technology: Critical Insights. International Music Education Research Centre, iMerc. Institute of Education University of London.

Housand, B.C. y Housand, A.M. (2012). The role of technology in gifted students' motivation. Psychology in the Schools, 49(7), 706-715.

Hwang, G.J. et al. (2012). Development of a personalized educational computer game based on students' learning styles. Educational Technology Research and Development, 60(4), August, 623-638.

Irigoien, I. y Arenas, C. (2006). Clasificación: Análisis de clusters (clustering). En B. Sierra (Coord.), Aprendizaje automático: conceptos básicos y avanzados. Aspectos prácticos utilizando el software Weka. Madrid: Pearson Prentice Hall.

Jorgensen, E. R. (2008). The art of teaching music. Indianápolis: Indiana University Press. Kearney, M.S. \& Levine, P.B. (2015). Early Childhood Education by MOOC: Lessons from Sesame Street. NBER Working, Paper No. 21229, June. (DOI: 10.3386/w21229).

Kop, R. (2011). The challenges to connectivist learning on open online networks: Learning experiences during a massive open online course. The International Review of Research in Open and Distance Learning, 12(3), 19-38.

Lin, C.F. et al. (2013). Data mining for providing a personalized learning path in creativity: An application of decision trees. Computers \& Education, 68, October, 199-210.

Lin, Y. et al. (2012). An improved clustering method based on k-means. Paper presented in 9th International Conference on Fuzzy Systems and Knowledge Discovery (FSKD), Sichuan, 29-31 May, 734-737. DOI: 10.1109/FSKD.2012.6234296.

Mairal, J. et al. (2010). Online learning for matrix factorization and sparse coding. Journal of Machine Learning Research, 11, 19-60.

McAfee, A. et al. (2012). Big Data. The management revolution. Harvard Business Review, 90(10), 61-67. 
Monedero-Moya, J.-J., Cebrián-Robles, D. y Desenne, Ph. (2015). Usabilidad y satisfacción en herramientas de anotaciones multimedia para MOOC. Comunicar, 44(XXII), 55-62. DOI: http://dx.doi.org/10.3916/C44-2015-06

Montgomery, A. et al. (2015). Blending for student engagement: Lessons learned for MOOCs and beyond. Australasian Journal of Educational Technology, 31(6), 657670.

Moodle (2016). Modular Object-Oriented Dynamic Learning Environment. Recuperado el 10 de Febrero de 2016 de http://www.moodle.org

Pang, J., Jie, L. \& Xu, F. (2014). Study on the Group Cooperative Innovation Based on WEB2.0. Journal of Software, 9(3), 613-620.

RAND Corporation (2014). Early progress: Interim research on personalized learning. Seattle, WA: The Bill and Melinda Gates Foundation.

Redding, S. (2014a). Personal Competencies in Personalized Learning. Center on Innovations in Learning, Temple University, Philadelphia, PA. http://www.centeril.org/publications/Personalized_Learning.pdf (16-05-2016).

Redding, S. (2014b). Personal Competency. A Framework for Building Students' Capacity to Learn. Philadelphia, PA: Temple University, Center on Innovations in Learning. http://www.centeril.org/publications/Personal_Compentency_Framework.pdf (1605-2016).

Redding, S. (2014c). The something other: Personal competencies for learning and life. Philadelphia, PA: Temple University, Center on Innovations in Learning. http://eric. ed.gov/?id=ED558074 (16-05-2016).

Rivera, Claudia (2014). Objetos de aprendizaje: una primera Mirada . Infotecarios. http:// www.infotecarios.com/objetos-de-aprendizaje-una-primera-mirada/

Roberts-Mahoney, H., Means, A.J. \& Garrison, M.J. (2016). Netflixing human capital development: personalized learning technology and the corporatization of K-12 education. Journal of Education Policy. DOI: http://dx.doi.org/10.1080/02680939.2015. 1132774

Sadovaya, V.V.; Korshunova, O.V. \& Nauruzbay, Z.Z. (2016). Personalized Education Strategies. Mathematics Education, 11(1), 199-209.

Sagitova, R.R. (2014). Training students to be autonomous learners. International Journal of Humanities Education, 12(1), 27-34.

Sandler, S. (2012). People v. 'personalization': Retaining the human element in the hightech era of education. Education Week, 31(22), 20-22.

Selwyn, N. (2012). Ten suggestions for improving academic research in education and technology. Learning, Media and Technology, 37(3), 213-219.

Sharif, A., \& Magrill, B. (2015). Discussion forums in MOOCs. International Journal of Learning, Teaching and Educational Research, 12(1), 119-132.

Shechtman, N. et al. (2013). Promoting grit, tenacity, and perseverance: Critical factors for success in the 21st century. Washington, DC: U.S. Department of Education, Department of Educational Technology.

Spector, J.M. (2013). Emerging Educational Technologies and Research Directions. Journal of Educational Technology \& Society, 16(2), April, 21-30.

Tseng, S.-F. et al. (2016). Who will pass? Analyzing learner behaviors in MOOCs. Re- 
Evaluación de objetos digitales de aprendizaje musical en Moodle

Manuel Jesús Espigares Pinazo y José Manuel Bautista Vallejo

search and Practice in Technology Enhanced Learning, 11(8), 1-11. DOI: 10.1186/ s41039-016-0033-5

Twyman, J. (2014). Competency-based Education: Supporting Personalized Learning. Philadelphia, PA: Center on Innovations in Learning. http://www.centeril.org/connect/ resources/Connect_CB_Education_Twyman-2014_11.12.pdf (16-05-2016).

Vollenbroek, W. et al. (2014). Learning Education: An 'Educational Big Data' approach for monitoring, steering and assessment of the process of continuous improvement of education. Paper presented in European Conference in the Applications of Enabling Technologies, 20-21 November 2014, Glasgow, Scotland.

Wolf, M. (2010). Innovate to education: System [re]design for personalized learning. A report from the 2010 symposium. Washington, DC: Software \& Information Industry Association. Retrieved from http://siia.net/pli/ presentations/PerLearnPaper.pdf (1605-2016).

Yang, S. et al. (2011). A collaborative multimedia annotation tool for enhancing knowledge sharing in CSCL. Interactive Learning Environments, 19(1), 45-62. DOI: 10.1080/10494820.2011.528881 
Anexo

\section{TEST DE EVALUACIÓN INICIAL PRIMERO DE LA ESO}

\section{NOMBRE Y APELLIDOS: CURSO Y GRUPO:}

1. Escribe en un pentagrama las figuras y los silencios de: negra, corchea y semifusa. 2 PUNTOS

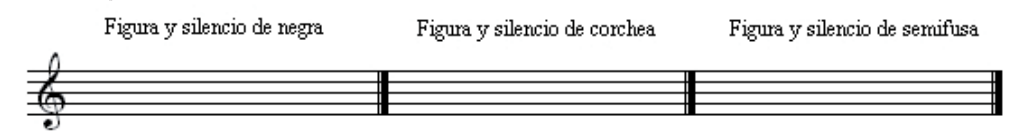

2. Indica cuántos tiempos tiene una redonda y una blanca. 1 PUNTO

3. ¿Qué efecto tiene el sostenido? Represéntalo. 1 PUNTO

4. Completa el siguiente pentagrama en clave de sol y en compás de cuatro por cuatro con la figura o las figuras que falten. 1 PUNTO

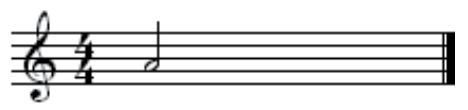

5. Realiza la clasificación de los instrumentos musicales y pon ejemplos de cada familia. 1 PUNTO

6. Señala los tipos de compases que puede tener una audición. 1 PUNTO

7. Realiza este test sobre el nombre de las notas en clave de sol y clave de fa en $4^{\circ}$ línea de la siguiente página. 3 PUNTOS.
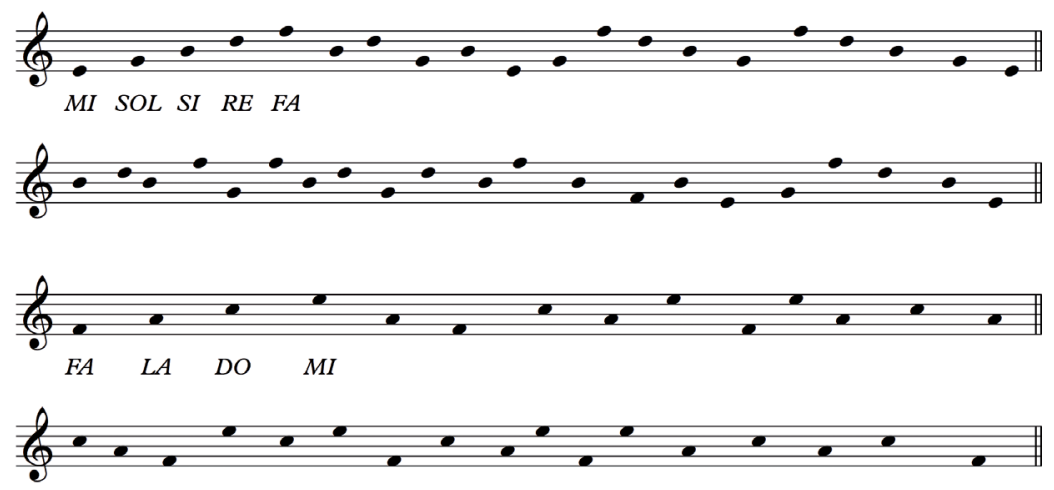
Evaluación de objetos digitales de aprendizaje musical en Moodle

Manuel Jesús Espigares Pinazo y José Manuel Bautista Vallejo
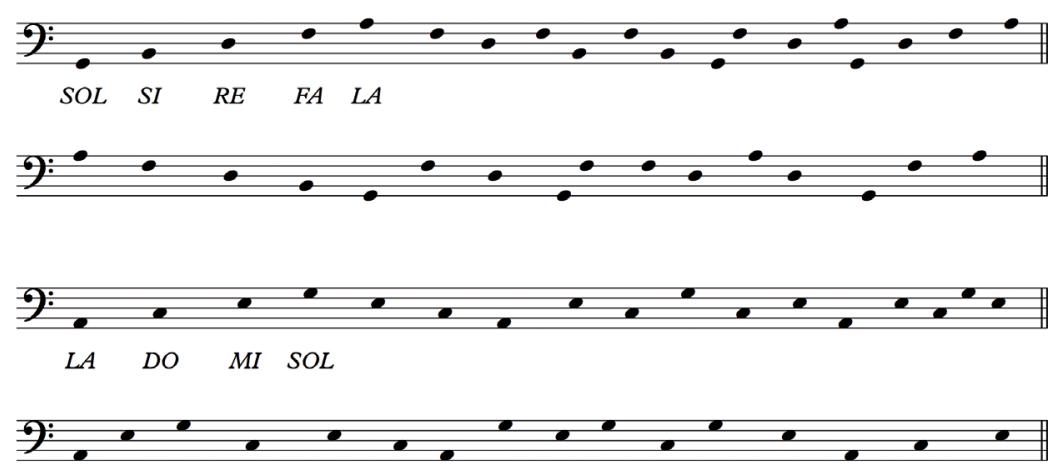